\title{
Value of the Serum Galectin 3 in Patients with Hepatocelluar Carcinoma: A Meta-analysis \\ L Zhang ${ }^{1}$, X-J Chen ${ }^{2}$, D He ${ }^{3}$, T-Y Zhou ${ }^{3}$
}

\begin{abstract}
Purpose: To investigate the connection of galectin 3 with HCC risk.

Methods: Publications were searched using PubMed, MEDLINE, EMBASE and the Chinese databases (including CNKI and WanFang) up to October 2015.

Results: A total of four studies were included into this analysis. The combined mean difference suggested that serum Galectin 3 levels tended to be associated with hepatitis $1.98(95 \%$ CI: $1.13-2.83, \mathrm{Z}=4.57, \mathrm{P}<0.00001)$ and healthy person 2.29(95\% CI: 2.09-2.5, Z=21.78, $\mathrm{P}<0.00001$ ). The serum Galectin3 level in HCC was significant higher than that in hepatitis and healthy person. The pooled sensitivity and specificity were 0.93 (95\% CI: $0.86-0.97$ ) and 0.83 (95\% CI: 0.74-0.90), the pooled DOR were 116.78 (95\% CI: 0.13-102122.46), the pooled positive LR were 12.71 (95\% CI: 0.12-1374.27), and the pooled negative LR were 0.11 (95\% CI: 0.00-12.51).
\end{abstract}

Conclusion: The serum Galectin 3 level of HCC is higher than that of hepatitis and healthy person. And the expression of serum Galectin 3 tended to be a bigger possibility biomarker for diagnosis of HCC.

Keywords: Hepatocelluar carcinoma, meta-analysis, serum Galectin

From: Department of Infectious Diseases, West China Hospital, Si Chuan University, Cheng Du, Si Chuan, P.R. China.

Correspondence: Dr T-Y Zhou, Department of Infectious Diseases, West China Hospital, Si Chuan University, Si Chuan PR China. E-mail: 18580512362@163.com 


\section{INTRODUCTION}

Hepatocellular carcinoma (HCC) is the sixth most common cancer, which is attributed to the third cause of cancer-related mortality in overall global(1). Prognosis of hepatocelluar carcinoma is very poor with a just $14 \%$ five-year survival rate because of the rapid process and high malignancy (2, 3). Lacking of novel biomarkers, HCC is frequently found in late stages. Commonly, we use CT, ultrasound and MRI and blood chemistry tests to diagnosis HCC. But histopathologic assessment is necessary, when the imaging tests and blood chemistry tests are atypical. However, it is a problem for the doctor to do the invasive approach and get the right tissues from patients with hepatocelluar carcinoma. So it is an interesting thing that we can find new serum biomarkers to evaluate the diagnostic efficiency of hepatocelluar carcinoma. In clinical, serum alpha-fetoprotein (AFP), with low sensitivity, is used to diagnosis the HCC(4). However, a new serum biomarker needs to be found and to be improved or even to be replaced the performance of AFP which is not satisfactory.

Galectins are a growing family of b-galactoside-binding animal lectins, composed of 2 domains: a carboxylterminal domain and an amino-terminal domain (5). Many studies suggest that galectin-3, a unique chimera-type member of the b-galactoside-binding soluble lectin family, has been proposed to have multifaceted functions including cell growth, proliferation, adhesion, differentiation, immune responses, angiogenesis, apoptosis, metastasis and tumor progression(68). The expression of Galectin 3 is a promising diagnostic indicator for kinds of carcinomas, such as carcinoma of stomach(9), colon carcinoma(10)and thyroid carcinoma(11). However, the relation between galectin 3 and $\mathrm{HCC}$ risk is unclear. Our targets are to analysis the expression of 
serum galectin-3 in different stages of liver diseases and evaluate the relationship between diagnosis and serum galectin-3 expression in HCC patients.

\section{SUBJECTS AND METHODS}

\section{Search strategy}

The publications were searched in PubMed, MEDLINE, EMBASE and the Chinese databases (including CNKI and WanFang database ) until October 2015 by using the following search terms:(hepatocelluar carcinoma or liver cancer or liver cell carcinoma or HCC)AND(Galectin 3 or gal-3).

\section{Study selection}

Studies in this meta-analysis meeting the criteria as the following:

Original articles directly explored the serum Galectin 3 expression in different stages of diseases (including HCC, hepatic cirrhosis, hepatitis, or healthy person);

Original articles directly explored the diagnostic performance of serum Galectin 3 for HCC;

Using ELISA to examine the serum Galectin 3 expression;

Sufficient information were reported to estimate an mean difference (MD),95\% confidence interval (CI), true positive (TP), false positive(FP), false negative (FN)and true negative (TN).

Studies were excluded as following: (1) case reports, reviews, letters and editorial articles. (2) articles in which sufficient datum were not contained or calculated were also excluded. 


\section{Data extraction}

Two independent investigators (L.Z and DH) extracted all data from eligible studies to minimize the bias, a third author (XJC) resolved disagreements through discussion. For each article, the following characteristics were recorded: the first author, the publication year, number of patients, the test method $\mathrm{TP} 、 \mathrm{FP} 、 \mathrm{FN} 、 \mathrm{PN}$ and cut-off value.

\section{Statistical analysis}

All analysis was performed by Review Manager 5.3 and MetaDisc. We extracted data including serum Galectin 3 levels of HCC versus serum Galectin 3 levels of hepatic cirrhosis, hepatitis, and healthy person in each article. Mean difference and 95\%CI were applied to provide the effective value. And in order to evaluate the diagnostic efficiency of serum Galectin 3 in hepatocelluar carcinoma, we also extracted the TP、 FP、FN and PN to estimate the sensitivity and specificity. Initially we retrieved 191 studies from PubMed, Embase the Chinese databases (including CNKI and WanFang database) up to October 2015.

Scanning the titles and abstracts, 181 were excluded, because they were reviews, experimental research, reports, duplicate articles or not relevant to the current serum Galectin 3 in HCC. After reading the remaining 10 full -text articles, 6 were excluded, because they used other test methods but not ELISA. Finally four articles were included for conducting with meta-analysis ( seen in Figure 1). Totally aiming to assess the serum Galectin3 expression, 120 patients with HCC, 68 patients with hepatic cirrhosis, 47 patients with hepatitis and 24 healthy person in these 4 articles were used to explore the serum Galectin 3 levels in different stages of diseases. What is 
more, we also acquired the TP、 FP、 FN and PN to estimate the sensitivity and specificity of hepatocelluar carcinoma by detecting the serum Galectin3 expression in HCC and non-tumor. ELISA was used to detect the serum Galectin 3. A random effect model for $\mathrm{I}^{2}>50 \%$, a fixed effect model for $\mathrm{I}^{2}<50 \%$, statistical significance from different studies was defined as a P value less than 0.05(12). (Table1 and table 2)

\section{RESULTS}

In the 4 studies, the meta analysis results: Mean difference [0.63(95\% CI:-0.61$1.87, \mathrm{Z}=0.99, \mathrm{P}=0.325)]$ indicated that there was no significant differences for serum Galectin 3 level between HCC and hepatic cirrhosis with an obvious heterogeneity (Tau-squared=0.92, chisquared $=9.83, \mathrm{I}^{2}=80 \%, \mathrm{P}=0.007$ ) (figure $2 \mathrm{~A}$ ). However, we also found the serum Galectin 3 level in HCC was significant higher than that in hepatitis and healthy person. The pooled mean difference for $\mathrm{HCC}$ versus hepatitis was [1.98(95\%CI: 1.13-2.83, $\mathrm{Z}=4.57, \mathrm{P}<0.00001)]$ without the heterogeneity (chi-squared $=0.21, \mathrm{I}^{2}=0 \%, \mathrm{P}=0.64$ ) (figure2B), The pooled mean difference for HCC versus healthy person was [2.29(95\%CI: 2.09-2.5, $\mathrm{Z}=21.78, \quad \mathrm{P}<0.00001)]$ without heterogeneity (chi-squared $\left.=0.07, \mathrm{I}^{2}=0 \%, \mathrm{P}=0.79\right)$ (Figure2C).

In our analysis ,a total $110 \mathrm{HCC}$ patients and 111 non-tumor patients were conducted this meta-analysis to evaluate the diagnostic value of serum Galectin3.The overall sensitivity and specificity were 0.93 (95\% CI: 0.86-0.97) and 0.83 (95\% CI: 0.74-0.90), the pooled DOR were 116.78 (95\% CI: 0.13-102122.46), the pooled positive LR were 12.71 (95\% CI: 0.12-1374.27), 
and the pooled negative LR were 0.11 (95\% CI: 0.00-12.51). The all results indicated that galectin 3 may be a useful biomarker for diagnosis of HCC. (Figure 3)

\section{DISCUSSION}

Prognosis of hepatocelluar carcinoma is very poor because of the rapid process and high malignancy. Radiotherapy, chemotherapy and surgery are the main treatment methods for HCC patients in clinic. Despite the therapies have been improved, the curative effect is still poor. Therefore, it is urgent to engage in finding potent diagnosis factors to diagnosis HCC patients.

In our study, we enrolled 4 studies to derive a more precise estimation on the serum Gal3 levels of HCC patients versus serum Gal-3 levels in hepatic cirrhosis, hepatitis, and healthy person. And we also estimated the diagnostic effect of the serum Gal-3 for HCC. The results indicated serum Galectin3 level in HCC was significant increased compared to those in hepatitis and healthy person suggested galectin3 played an important role in the pathogenesis of the hepatocelluar carcinoma. But there was no significant difference for serum Galectin 3 level between HCC and hepatic cirrhosis. Indeed, Daniel K in the study of "galectin 3 experssion is induced in cirrhotic liver and hepatocellular carcinoma" said galectin-3 was abundantly expressed in cirrhotic liver in peripheral distribution within regenerating nodules. Such galectin-3 expression in rapidly proliferating hepatocytes in cirrhotic liver may be a result of the high mitotic index. Alternatively, it is possible that proliferating cells expressing galectin-3 are in the process of being transformed, thus may indicate an early neoplastic event. It indicated higher galectin 3 level may 
be a potential biomarker for disease diagnosis. The meta-analysis showed that the overall sensitivity and specificity were 0.93 and 0.83 , which had a certain clinical value to evaluate the diagnosis of HCC, used as an auxiliary diagnostic method. The pooled DOR (Diagnostic odds ratio) reflects the accuracy of diagnostic tests as a reliable indicator, the greater its value indicates the stronger the diagnostic ability to distinguish. In our study the pooled DOR were 116.78 , suggested the efficiency of diagnosis relatively high. The pooled positive LR were 12.71 , prompted the positive rate of detection of HCC was 12.71 times more than non-HCC.

The pooled negative LR implied once the detection of galectin 3 was negative, the risk of liver cancer is $11 \%$. Overall, full consideration of the clinical symptoms, combined with serum galectine 3, is helpful for early diagnosis. Zhou's study(4) included eight studies for serum AFP in diagnosing $\mathrm{HCC}$ were as follows: sensitivity $70 \%$; specificity $89 \%$; diagnostic odds ratio (DOR) 18.00(9.41-34.46), which we can indicate that serum Galectin 3 may be a more useful diagnostic biomarker for HCC. The problem in our analysis should be pointed out: it had no significant association between the serum galectin 3 levels and hepatic cirrhosis with an obvious heterogeneity. However, we did not analysis the publication bias, because the studies we internalized were just three.

\section{CONCLUSION}


Our meta-analysis indicates that galectin3 played an important role in the pathogenesis of the hepatocelluar carcinoma. And also may have a better diagnostic accuracy for making the diagnosis of HCC. More studies should be addressed to evaluate the diagnostic accuracy of Gal-3.

\section{ACKNOWLEDGEMENTS}

Thank the support and help of the all members in the Department of Infectious Diseases.

\section{AUTHORS' NOTE}

Lu Zhang collected data and wrote manuscript and approved final version. Xiang-Jun Chen conducted data analysis, and approved final version. Da He looked for the papers and collected the useful data and approved final version, Tao-You Zhou critically revised manuscript and approved final version. The authors declare that they have no conflicts of interest. 


\section{REFERENCES}

1. Jemal A, Bray F, Center MM, Ferlay J, Ward E, Forman D. Global cancer statistics. CA: a cancer Journal Clinicians 2011; 61: 69-90.

2. Yamashita T, Wang XW. Cancer stem cells in the development of liver cancer. J Clin Investigat $2013 ; \mathbf{1 2 3}: 1911-8$.

3. Cao K, Lu C, Han S, Zou Q, Li J, Xie D, et al. Expression of Girdin in primary hepatocellular carcinoma and its effect on cell proliferation and invasion. Int J Clin Exp Pathol 2015; 8: 551-9.

4. Zhou Y, Yin X, Ying J, Zhang B. Golgi protein 73 versus alpha-fetoprotein as a biomarker for hepatocellular carcinoma: a diagnostic meta-analysis. BMC Cancer 2012; 12: 17.

5. Nita-Lazar M, Banerjee A, Feng CG, Vasta GR. Galectins regulate the inflammatory response in airway epithelial cells exposed to microbial neuraminidase by modulating the expression of SOCS1 and RIG1. Mol Immunol 2015; 68: 194-202.

6. Nangia-Makker P, Balan V, Raz A. Regulation of tumor progression by extracellular galectin-3. Cancer Microenvironment 2008; 1: 43-51.

7. Liu FT, Patterson RJ, Wang JL. Intracellular functions of galectins. Bba-Gen Subjects 2002; 1572: 263-73.

8. Liu FT, Rabinovich GA. Galectins as modulators of tumour progression. Nat Rev Cancer 2005; 5: 29-41.

9. Lotan R, Ito H, Yasui W, Yokozaki H, Lotan D, Tahara E. Expression of a 31-kDa 
lactoside-binding lectin in normal human gastric mucosa and in primary and metastatic gastric carcinomas. Int J Cancer 1994; 56: 474-80.

10. Schoeppner HL, Raz A, Ho SB, Bresalier RS. Expression of an Endogenous GalactoseBinding Lectin Correlates with Neoplastic Progression in the Colon. Cancer 1995; 75: 2818-26.

11. Cvejic D, Savin S, Golubovic S, Paunovic I, Tatic S, Havelka M. Galectin-3 and carcinoembryonic antigen expression in medullary thyroid carcinoma: possible relation to tumour progression. Histopathology 2000; 37: 530-5.

12. Higgins JPT, Thompson SG. Quantifying heterogeneity in a meta-analysis. Stat Med 2002; 21: 1539-58.

13. Eisa NH, Ebrahim MA, Ragab M, Eissa LA, El-Gayar AM. Galectin-3 and matrix metalloproteinase-9: Perspective in management of hepatocellular carcinoma. J Oncol Pharmacy Practice : 2015; 21: 323-30.

14. Matsuda Y, Yamagiwa Y, Fukushima K, Ueno Y, Shimosegawa T. Expression of galectin3 involved in prognosis of patients with hepatocellular carcinoma. Hepatol Res 2008; 38: 1098-111.

15. Ulu M, Alacacioglu A, Yuksel E, Pamukk BO, Bozkaya G, Ari A, et al. Prognostic significance of serum galectin-3 levels in patients with hepatocellular cancer and chronic viral hepatitis. Saudi J Gastroenterol 2015; 21: 47-50.

16. Fang Q-q. Serum and tissue expressions of galectin-3 in hepatocelluar carcinoma and the clinical significances. Chin J Hepatol July 2011; 19: 527-31. 
Table 1: Serum galectin 3 levels in patients with HCC, cirrhosis, hepatitis and healthy people

\begin{tabular}{llllll}
\hline ref & year & HCC & Cirrhosis & hepatitis & $\begin{array}{l}\text { Healthy } \\
\text { people }\end{array}$ \\
\hline Nada (13) & 2015 & 50 & 30 & 0 & 10 \\
Yasunori (14) & 2008 & 51 & 16 & 23 & 14 \\
Mehmet (15) & 2015 & 19 & 22 & 24 & 0 \\
\hline
\end{tabular}

Table 2: Galectin 3 expression in HCC and non-tumor

\begin{tabular}{llccccccc}
\hline ref & year & TP & FP & FN & PN & Cut-off & $\begin{array}{l}\text { HCC/No } \\
\text { n-tumor }\end{array}$ & $\begin{array}{l}\text { Detect } \\
\text { method }\end{array}$ \\
\hline $\begin{array}{l}\text { Qing- } \\
\text { QingFa }\end{array}$ & 2011 & 40 & 1 & 1 & 89 & $0.62 \mathrm{ng} / 1$ & $62 / 90$ & ELISA \\
$\begin{array}{l}\text { ng(16) } \\
\begin{array}{l}\text { Yasuno } \\
\text { ri(14) }\end{array}\end{array}$ & 2008 & 34 & 7 & 14 & 14 & $\begin{array}{l}2.76 \mathrm{ng} / \mathrm{m} \\
1\end{array}$ & $48 / 21$ & ELISA \\
\hline
\end{tabular}

(True positive $=\mathrm{TP}$, false positive $=\mathrm{FP}$, false negative $=\mathrm{FN}$, true negative $=\mathrm{TN}$.) 


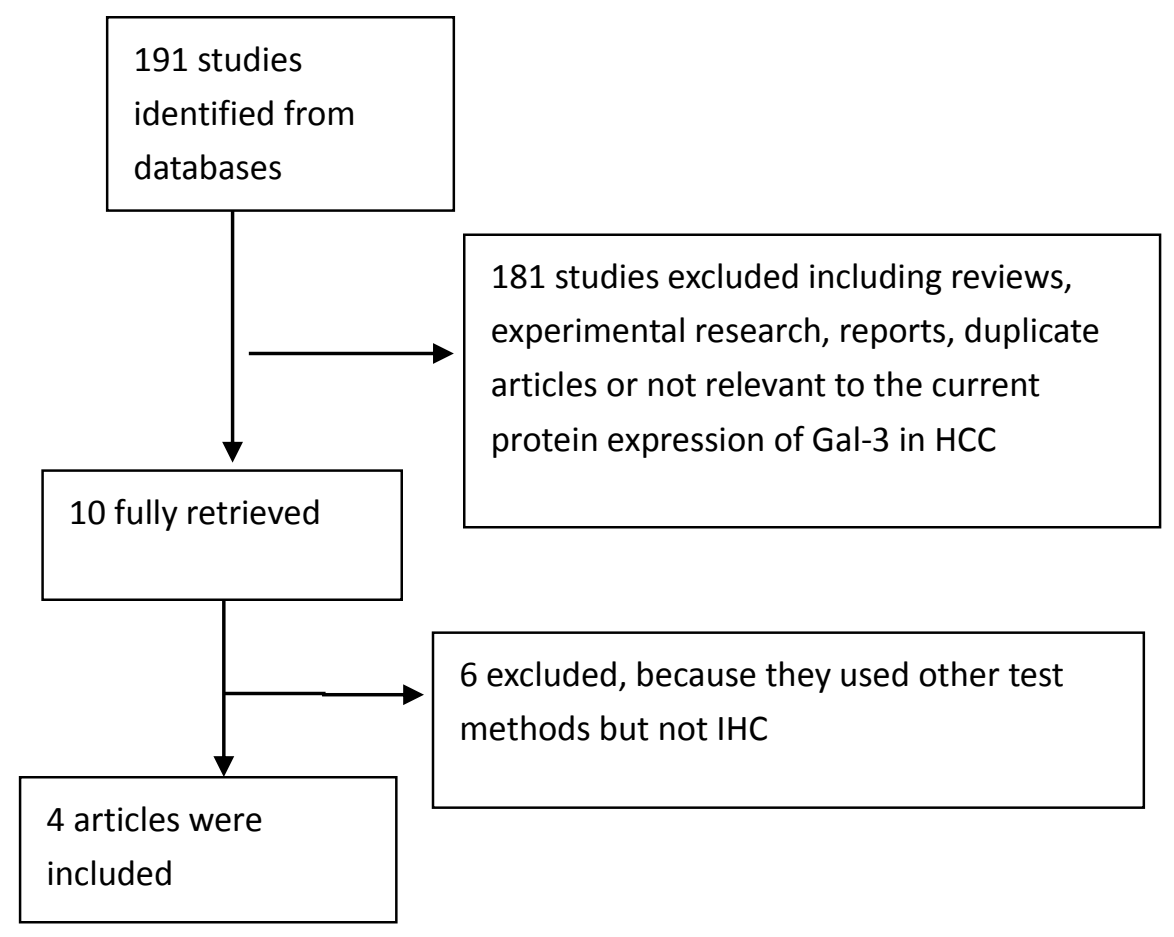

Fig.1: The study selection process 
Zhang et al

A

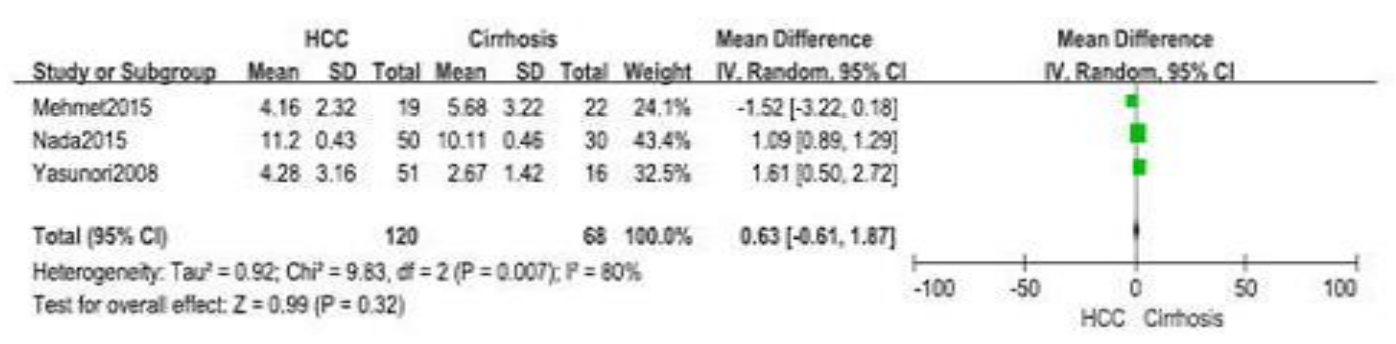

B

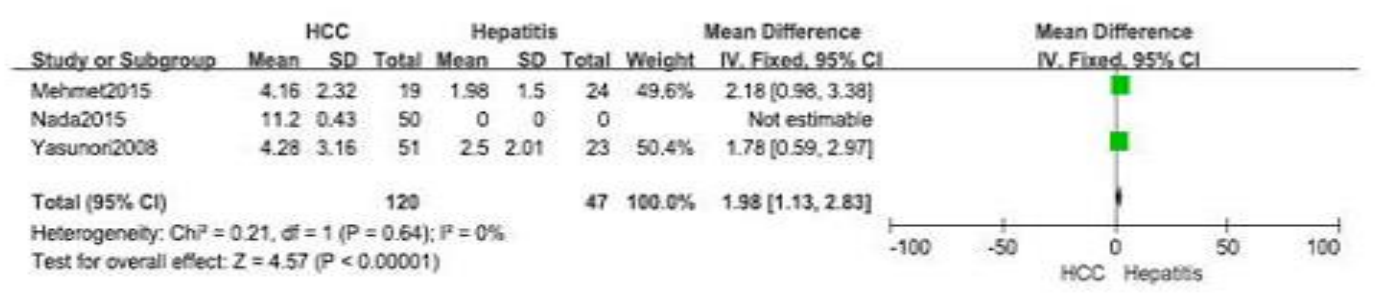

C

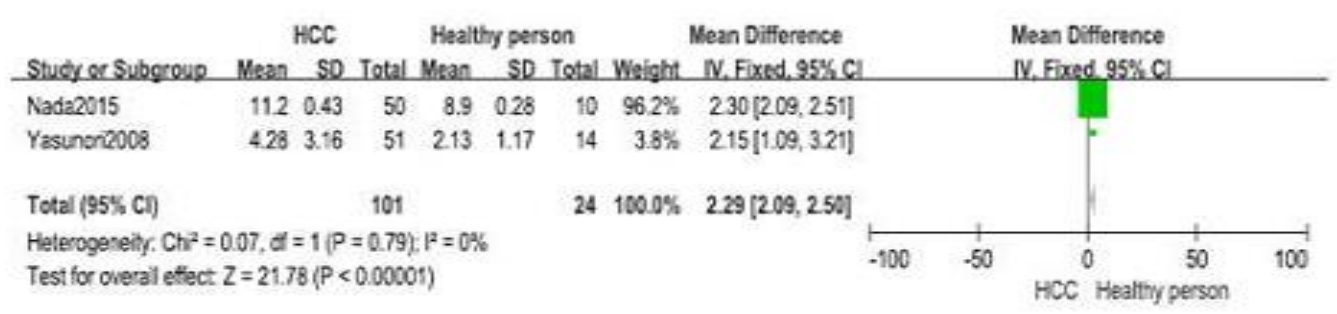

Fig. 2 (A): Forest plot for serum Galectin3 in HCC and Hepatic cirrhosis; (B): Forest plot for serum Galectin3 in HCC and Hepatitis; (C): Forest plot for serum Galectin3 in HCC and Healthy person. 
A

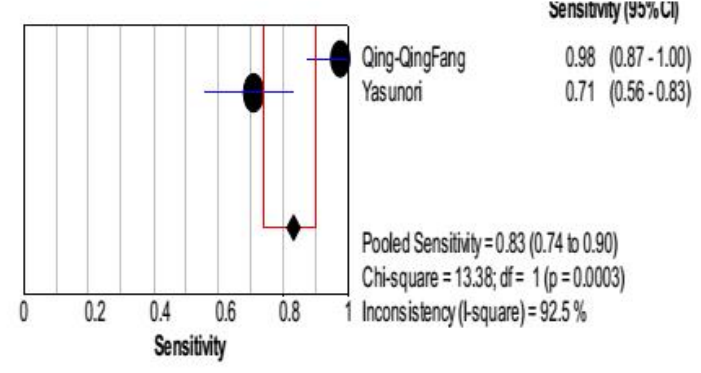

B

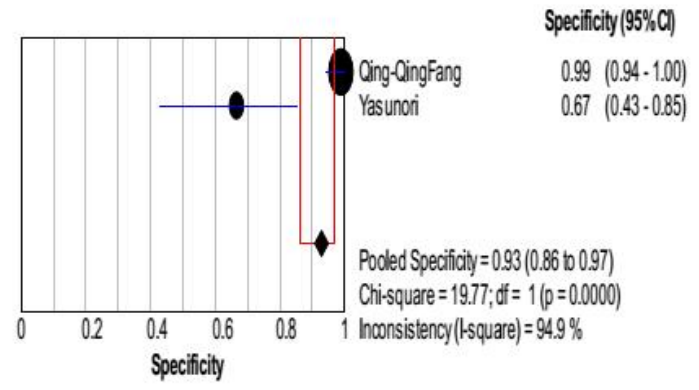

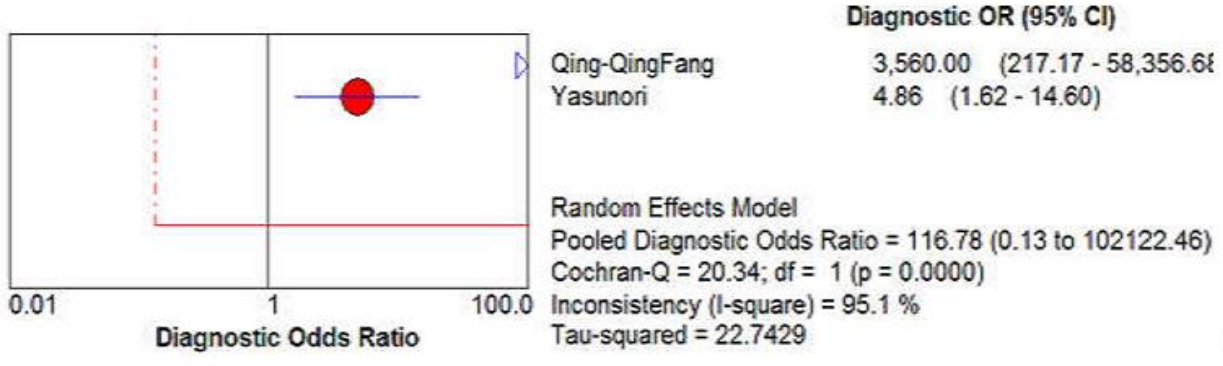

C

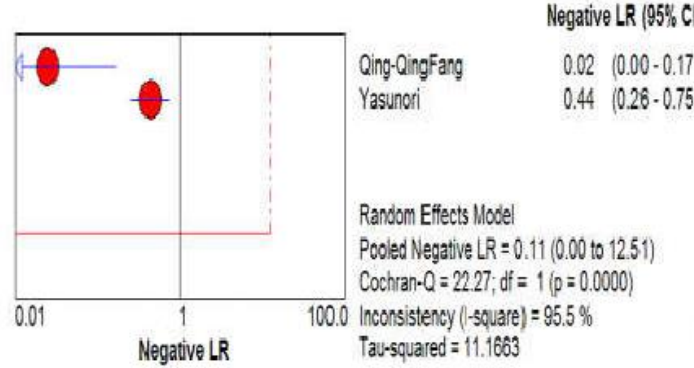

D

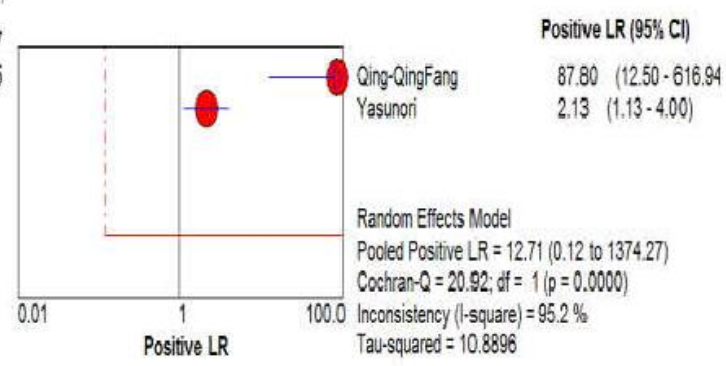

E

Fig.3(A): Forest plot of the sensitivity of serum Galectin 3 in HCC diagnosis; (B): Forest plot of the specificity of serum Galectin 3 in HCC diagnosis; (C): Forest plot of the DOR of serum Galectin 3 in HCC diagnosis. (D): Forest plot of the pooled negative LR of serum Galectin 3 in HCC diagnosis; (E): Forest plot of the pooled positive LR of serum Galectin 3 in HCC diagnosis. 